\title{
Isoelectric Focusing of Proteins in Rewetted Ultrathin Polyacrylamide Gel Layers ${ }^{1}$
}

\author{
THEODORE O. SIPPEL
}

Department of Anatomy and Cell Biology, The University of Michigan Medical School, Ann Arbor, Michigan 48109

Received May 19, 1986

\begin{abstract}
Ultrathin layers of polyacrylamide gel bound to glass can be washed, air-dried, and stored for at least 1 year before rewetting in ampholyte solutions for isoelectric focusing. Short-term drying affects neither fluorescent banding of the ampholytes (not evident in conventional gels) nor resolution of complex protein mixtures while prolonged storage seems to have no deleterious effects. Layers are fully functional after soaking for $10 \mathrm{~min}$ in solutions that may contain $8 \mathrm{M}$ urea and $10 \%$ sorbitol. Rewetting allows the rapid survey of different ampholytes, gradient stabilizers, separator compounds, or protein reagents and is adaptable to concentration modification of the $\mathrm{pH}$ gradient (alone or with a gel overlay), to focusing in a transverse urea gradient, and to electrophoresis across a preformed $\mathrm{pH}$ gradient. The procedure avoids protein modification by residual polymerizing reagents while adding to the convenience and economy of using ultrathin layers in relatively small formats. (c) 1986 Academic Press, Inc.

KEY WORDS: isoelectric focusing; electrophoresis; ultrathin polyacrylamide gel; rewetting; structural proteins; hemoglobins.
\end{abstract}

Eckersall and Conner (1) noted distorted $\mathrm{IEF}^{2}$ patterns after using enough ammonium persulfate to produce firm ultrathin layers of polyacrylamide gel bound to polyester sheets. To get around the difficulty they devised a procedure in which ampholytes were infused into washed gels but found that at least a week was necessary for adequate equilibration. However, Robinson (2) previously obtained satisfactory focusing in relatively thick washed layers that were soaked in ampholyte solutions for only $6 \mathrm{~h}$ after thorough drying. This approach recently has been rediscovered in various forms (3-5) and independently in the study described here. A methodology involving ultrathin gels bound to either glass or polyester supports in semi-miniature formats is presented and its applicability to several IEF techniques demonstrated. Analysis of newt

\footnotetext{
' Work supported in part by Grant EY05419 from the National Eye Institute (to T. G. Connelly).

${ }^{2}$ Abbreviations uscd: DTE, dithiocrythritol; IEF, isoelectric focusing.
}

lens proteins (crystallins) using the system is underway as part of a collaborative project on lens regeneration.

\section{MATERIALS AND METHODS}

Supplies and equipment. Materials, identified below by source, were purchased from Aldrich Chemical Company (Milwaukee, Wisc.), FMC BioProducts (Rockland, Maine), Serva Fine Biochemicals (Westbury, N.Y.), and Schwarz/Mann (Orangeburg, N.Y.). Dimethyldichlorosilane and Aqua-sil (mainly an octadecyltrialkoxysilane) were obtained from the Pierce Chemical Company (Rockford, Ill.) and applied as directed. Other compounds, except common reagents, were from Sigma Chemical Company (St. Louis, Mo.). Supplies such as $50 \times 75 \times 1.2-\mathrm{mm}$ microslides (Clay Adams Gold Seal; Becton-Dickinson Co., Parsippany, N.J.) were available from laboratory supply houses. Artist's acetate film and a selection of waterproof polyethylene (sealing) and vinyl (electrical) tapes were bought locally. 
Two prototype apparatuses for semi-miniature IEF (Ann Arbor Plastics, Inc., Ann Arbor, Mich.) were used with a 2000-V power supply (EC300; E-C Apparatus Corp., St. Petersburg, Fla.) in series with a supplemental O- to 1-mA meter. Rhodamine B fluorescence was measured in an assembled microscope fluorometer (6) and ampholyte fluorescence visible under two 4-W uv lamps (General Electric F4T5BLB) was photographed with transmitted excitation (Corning 7-60 primary and 3-144 secondary filters).

Proteins. Solutions were prepared in glassdistilled water: Protein test mixture 9 and $A n$ cistrodon rhodostoma venom (Serva) were 100 and $40 \mathrm{mg} / \mathrm{ml}$, respectively; frozen lenses of the adult eastern spotted newt, Notophthalmus viridescens, were homogenized in batches of 10 per $50 \mu \mathrm{l}$; and unit volumes of fingertip heparinized normal whole blood or the equivalent in saline-washed erythrocytes were lysed in 9 vol of $0.01 \% \mathrm{KCN}$. The preparations were centrifuged for $1 \mathrm{~min}$ at $17,000 \mathrm{~g}$ and stored at $4^{\circ} \mathrm{C}$ (hemolysates) or frozen.

Ultrathin layers. The monomer solution ( 20 ml) for each batch of gels was made with freshly dissolved reagents (all from Serva) and contained $970 \mathrm{mg}$ acrylamide (4X recrystallized grade), $30 \mathrm{mg} N, N^{\prime}$-methylenebisacrylamide $(2 \times)$, and $5 \mu \mathrm{l} N, N, N^{\prime}, N^{\prime}$-tetramethylethylenediamine. It was degassed with a water aspirator for $1 \mathrm{~min}$ before and $15 \mathrm{~s}$ after the addition of $100 \mu \mathrm{l}$ of fresh $5 \%$ ammonium persulfate; polymerization of samples began in 6-8 $\mathrm{min}$.

Layers approximating $40 \times 70 \times 0.1 \mathrm{~mm}$ were cast within 3 min onto microslides that had been treated at least 1 day earlier with fresh $0.2 \%(\mathrm{v} / \mathrm{v})$ 3-(trimethoxysilyl)propylmethacrylate (Aldrich) in undried acetone (cf. 7). Double molds were made by interposing between each pair of silanized slides a gel cover, comprised of a clean slide with a dried coating of a household liquid dishwashing detergent (e.g., Ajax, Joy, Sunlight) diluted 1: 2000 in water, and then inserting longitudinal spacers of nominally $5-\mathrm{mil}(0.13 \mathrm{~mm})$ acetate film that had been bent and sliced on a twoblade jig into $1 \times 5$-mm L-shaped cross sections for precise placement. Ten such sandwiches, interleaved with glassine powder paper to prevent polymerization on the backs, were stacked in a boat that permitted firm clamping between 1-cm-thick Plexiglas plates having slightly elevated rails to put pressure only over the spacers. After the molds were filled by capillarity, the boat was inverted flat to drain excess solution. Similar layers were cast singly on GelBond PAG (FMC) or Gel-Fix for PAGE (Serva) polyester sheets that were cut to 55 $\times 85 \mathrm{~mm}$ and flattened on wet glass.

After polymerizing for $2 \mathrm{~h}$ at room temperature in a moist chamber, the gels were washed with shaking in three changes of water over $30 \mathrm{~min}$ before all but a few on the glass were air-dried for culling and storage. Servalyt pH 3-10 Precote layers (Serva) scraped to 70$\mathrm{mm}$ lengths and $40-$ or $70-\mathrm{mm}$ widths were washed and dried in the same way.

Rehydration studies. To follow the course of gel swelling in various media, cross-section thicknesses of the layers immersed in relatively large volumes were measured under the microscope ( $10 \times$ objective) with an eyepiece micrometer. Uncertainty in the measurements because of optical refraction was not more than $5 \mu \mathrm{m}$. The dried gels broke sharply when the slides to which they were bound were scored on the back with a diamond point and snapped into fragments; undried gels were first sectioned to the glass with a razor blade.

The equilibration of a model solute under normal rewetting conditions (below) was determined by measuring the imbibition of 0.1 mM rhodamine B chloride (Aldrich). After the solution was advanced on a glass-bound layer for different cumulative intervals, the gel was blotted and redried for reading of fluorescence. Staining of the gel was negligible: the dye, unlike several others tested, did not become visibly more concentrated in the layer than in the bathing solution; and only about $0.4 \%$ of the initial fluorescence remained after fully equilibrated gels were shaken in water for 4 
min (about $0.1 \%$ would have been expected from the 24-s half-time for washout measured over the first $3 \mathrm{~min}$ ).

Ampholyte equilibration. The standard solution contained $2.5 \%(w / v)$ Servalyt of a specified range, $10 \%$ D-sorbitol, and $0.15 \%$ (10 mM) DTE. Urea (ultrapure, Schwarz/Mann) if present was freshly dissolved at $8 \mathrm{M}$ whereas separators (8) to be described were added from stock solutions before final dilution.

Routine rewetting of a dried layer was carried out under a thin Plexiglas cover elevated by two narrow strips of 0.15 - to 0.17 -mm-thick waterproof tape laid along opposite edges. About $0.6 \mathrm{ml}$ of the desired ampholyte solution pipetted onto the gel was spread without bubbles by sliding the cover into place and the assembly was held in a sealed container for 20 min or longer at room temperature. Just before use the gel was drained, blotted once with Whatman 50 (hardened) filter paper, and covered with a polyester film for transfer to the focusing apparatus.

If two portions of a gel were to be rewet separately, the first solution was slid to the boundary under a partial cover at least $20 \mathrm{~min}$ before similar application of the second. When this procedure was used to obtain a stepped difference in ampholyte concentration, i.e., for concentration modification of the $\mathrm{pH}$ gradient (9), the respective Servalyt levels were 10 and $2 \%$ in the otherwise standard mixture. "Concentration-thickness modification" utilized $2 \%$ Servalyt in the IEF gel and $10 \%$ in a short overlay, which was a layer of the usual thickness bound to polyester and cut to size before rewetting. For equilibrating dried and undried gels together in larger volumes, two slides with the layers facing were clamped on a $1.6-\mathrm{mm}$ thick silicone rubber gasket to make a cassette of 5-ml capacity; the same setup was used to rewet layers with a transverse urea gradient (below).

IEF procedure. In the apparatus designed for gels on microslides, recirculating water $\left(10^{\circ} \mathrm{C}\right)$ drawn through the base directly bathed the undersurface of the slide; the other model was similar except for having an alumina plate to which the polyester support holding a gel up to $10-\mathrm{cm}$ wide could be sealed with a few drops of water. While the covered layer was cooling, on either apparatus, filter papers positioned in endslots in the base were moistened with $\mathrm{N} \mathrm{NaOH}$, and electrode wicks (Serva) shortened to the width of the layer were soaked in $\mathrm{N}_{3} \mathrm{PO}_{4}$ as anolyte and a 4:1 (v/v) mixture of $\mathrm{N} \mathrm{NaOH}$ and $0.25 \mathrm{M} \mathrm{Ba}(\mathrm{OH})_{2}$ as catholyte. After the well-blotted wicks were placed 62 $63 \mathrm{~mm}$ apart on the gel, the apparatus lid carrying fixed platinum electrode wires against its lower surface was clamped to base supports previously adjusted for slight indentation of the wicks by the electrodes.

Focusing was begun at $1-\mathrm{mA}$ constant current and completed in $60-75 \mathrm{~min}$ at $2000 \mathrm{~V}$ without exceeding $2 \mathrm{~W}$. When approximately $1000 \mathrm{~V}$ was reached, samples imbibed in 1 mm-wide pieces of Whatman 50 paper $(0.3-$ $0.4 \mu \mathrm{l}$ for 5-mm lengths) or as comparably sized free droplets (Hamilton syringes) were placed $5 \mathrm{~mm}$ from the anode wick through a central hatch. The hatch cover was transparent to longwave uv light so that ampholyte fluorescence could be observed throughout focusing.

The gel was transferred to $15 \%$ trichloroacetic acid and 5\% sulfosalicylic acid within $15 \mathrm{~s}$ after opening of the circuit and then shaken for 5 min each in (a) this fixative, (b) $5 \%$ sulfosalicylic acid, (c) a 40 -fold dilution of $1 \%$ aqueous Coomassie brilliant blue G-250 (Serva) by the latter, giving a colloidal suspension stable for at least 1 week, and (d) $35 \%$ ethanol followed by drying in air.

Electrophoresis. The technique for displaying electrophoretic mobility as a function of $\mathrm{pH}$ in ultrathin gels (10) was adapted with little change to $70 \times 70-\mathrm{mm}$ reclaimed Servalyt Precote layers soaked in the standard ampholyte mixture ( $\mathrm{pH}$ 3-10 range). After focusing as above but without samples, scraping off of the wicks, and rotating the gel $90^{\circ}$ clockwise, a cotton thread soaked in the washed-cell hemolysate was stretched for $10 \mathrm{~s}$ across the 
gel midway between new wicks placed $62 \mathrm{~mm}$ apart. Electrophoresis for $15 \mathrm{~min}$ at $300 \mathrm{~V}$ was followed by the usual fixation and staining.

\section{RESULTS AND DISCUSSION}

The gels obtained were firm and adhered strongly to the silanized slides even if these had been stored for 10 weeks before use. Whereas patches commonly stuck to mold covers treated with dimethyldichlorosilane or coated by Kodak Photo-flo 200 (1:1000) and sometimes after treatment with Aqua-sil, slides coated with high dilutions of any one of six brands of liquid detergent consistently released the gels. No functional or physical consequences were detected in layers bound to glass although the less firmly adhering layers on polyester tended to be reticulated more frequently when cast under detergent-coated covers. Except where noted, the following studies were carried out on layers bound to slides. At the washed undried stage they generally measured $0.12-0.13 \mathrm{~mm}$ in thickness but sometimes were as thin as $0.11 \mathrm{~mm}$ in the centers despite attempts to avoid depressing the covers during casting.

Washed layers that had dried overnight were compared with their blotted undried counterparts to see whether drying affected the quality of focusing. Each pair was equilibrated for 24 $\mathrm{h}$ in $4.7 \mathrm{ml}$ of the standard ampholyte mixture (pH 3-10 range) strengthened slightly to allow for the calculated amount of water in the wet gel. Provided the solutions were withdrawn and reinjected repeatedly over the first few hours to assure the mixing needed for reproducible band spacing in the undried gels, seemingly identical patterns (not shown) were obtained with the test, venom, and newt lens proteins. The lack of any difference was more convincing, however, when the comparisons were made between the halves of individual layers in which the control sides were kept wet for $8 \mathrm{~h}$ before equilibration. IEF of the test protein mixture shown in Fig. la was a typical result. Perhaps because of the regular inclusion of $10 \mathrm{mM}$ DTE in the rewetting solutions, none of the three protein mixtures showed evidence of the oxidative (split band) artifact observed after drying of comparable gels (3). Although the demonstrated equivalence pertained to drying over relatively short terms, no changes in IEF characteristics have been noted in dried gels stored for 12 months.

In the preceding comparisons, bands of ampholyte fluorescence observed just before fixation were undeviated across the boundaries (Fig. 1b). While fluorescence was undetectable during focusing in some gels conventionally cast with ampholytes, presumably because of quenching by residual polymerizing reagents, the use of washed gels made it possible to monitor the successive formation, sharpening, and slight cathodal drift of patterns that were highly reproducible for each ampholyte range and consistent with the general staining patterns. Of the many light and dark bands best revealed by photography, the sharp bright pair tentatively estimated from Figs. 1a and $\mathbf{b}$ to lie at $\mathrm{pH} 7.65$ and 7.8 always was recognizable in situ even against fluorescing polyester supports; the more diffuse band at about $\mathrm{pH} 5.5$ usually could be identified as well. However, none of the bands could be equated to any particular element of the longwave uv pattern illustrated for $\mathrm{pH}$ 2-11 Servalyt in granular gels (11).

Further work was confined to dried gels. After immersion in water for $20 \mathrm{~min}$, adjacent glass-bound fragments that had dried overnight at room temperature or for another $5 \mathrm{~h}$ at $100^{\circ} \mathrm{C}$, i.e., under conditions of forced aging (4), regained identical thicknesses (measured in the same microscope field) equal within the error of measurement to those found before drying; the course of swelling was not followed. However in other air-dried samples stored 43 days, swelling in water appeared complete in 2-3 min and, when mixing was minimized to approximate routine rewetting conditions, only 3-4 min was required in the standard ampholyte mixture (pH 3-10 range). The presence of $8 \mathrm{M}$ urea in this solution delayed 

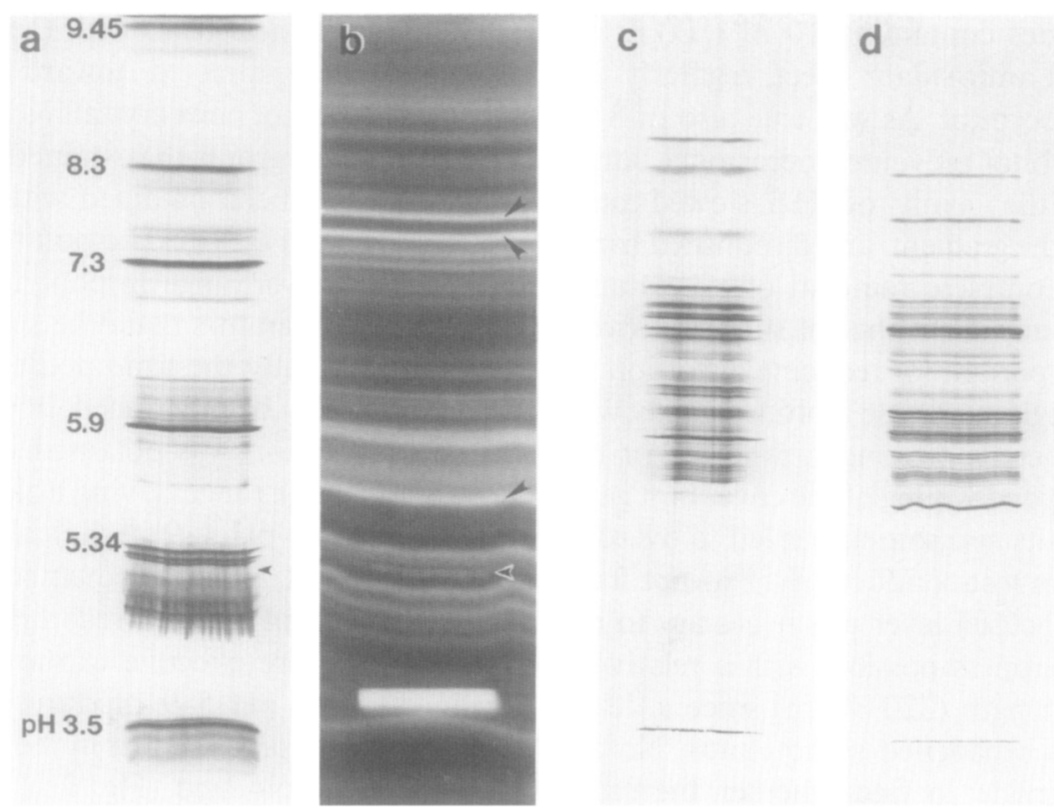

FIG. 1. IEF compared between half-layers equilibrated with pH 3-10 Servalyt; the 10-mm sample papers lie across the boundaries. Full interwick spans with cathodal ends up are shown in all figures. (a) Test mixture proteins in undried (left side) vs dried portions; an arrowhead indicates the position of atypical fluorescence similarly marked in (b), and the calibrations are $\mathrm{p} I$ values supplied by Serva. (b) Fluorescence in the untixed gel; inclined arrowheads locate bright bands described in the text. (c) Newt lens extract after rewetting for $24 \mathrm{~h}$ (left) and $10 \mathrm{~min}$. (d) The same extract after rewetting for $6 \mathrm{~h}$ (left) and $10 \mathrm{~min}$ by a solution containing $8 \mathrm{M}$ urea.

the process by at most another minute. Measuring rhodamine B imbibition showed the combined courses of swelling and the slower diffusion of a solute: despite considerable variability among trials, probably because some mixing could not be avoided during each advance of the solution over the dried gel, it was clear that equilibration was only $85-95 \%$ complete in $10 \mathrm{~min}$ although the dye content always stabilized by $20 \mathrm{~min}$.

Side-by-side comparisons then were made to determine how long dried gels actually had to be soaked in typical ampholyte solutions to give reproducible IEF patterns. The shortest period tested was $10 \mathrm{~min}$ which proved as effective as $24 \mathrm{~h}$ for the focusing of lens proteins in the standard mixture made up with $\mathrm{pH} 3-$ 10 Servalyt (Fig. 1c). Likewise, $10 \mathrm{~min}$ sufficed when the solution was supplemented with 8 M urea (Fig. 1d), but the control period now was limited to $6 \mathrm{~h}$ because of the slow breakdown of urea in solution; its instability at room temperature or, alternatively, crystallization in the cold prevented urea from being used at all in the prolonged soakings required for undried gels (1). Not illustrated are the similar results obtained when the comparisons were repeated with pH 5-9 Servalyt, both with and without urea, and the undeviated fluorescent banding that complemented the staining patterns in all cases. Observations made on thicker layers cast with immobilized $\mathrm{pH}$ gradients (12) suggest that the surprising rapidity with which gels exposed to strong urea solutions became functional may have arisen in part from limiting the volume of the rewetting solution to little more than the theoretical requirement.

Rewetting for subsequent applications was allowed to proceed for $20 \mathrm{~min}$ as a conservative minimum. The standard ampholyte so- 
lutions, besides containing $10 \mathrm{~mm}$ DTE for its presumed antioxidant effect, regularly included $10 \%$ sorbitol. As was true also of $8 \mathrm{M}$ urea, the sorbitol prevented occasional burning of gels in the vicinity of $\mathrm{pH} 5$, slowed cathodal drift of the gradient, and diminished band distortion. Complete removal of excess ampholyte solution from the gel surface proved especially important for reducing distortion in the anodal region; a single blotting usually was adequate whereas prolonged draining or repeated lifting and wiping of the cover film gave variable results and sometimes led to overdry gels that soon lost conductivity. Prompt fixation of the focused layer was necessary to realize the sharpness possible with a relatively high field strength $(320 \mathrm{~V} / \mathrm{cm})$ since a delay of only $30 \mathrm{~s}$ broadened some bands. No attempt was made to find whether the steps taken to lower ambient $\mathrm{CO}_{2}$ levels were necessary.
The first practical trials with the rewetting procedure were directed toward obtaining better separation of newt crystallins, neglecting the most acidic group that stained weakly if at all, than had been obtained with pH 3-10 ampholytes (cf. Fig. 1c). The point stressed is that both narrower range ampholyte mixtures and various separators could be surveyed serially in essentially the time needed to focus the layers. It may be mentioned, however, that the best resolution was obtained in $\mathrm{pH}$ 5-9 Servalyt (Fig. 2a); ranges giving little or no improvement were $\mathrm{pH}$ 4-9 (technical), $\mathrm{pH}$ 6-9, pH 7-9, and pH 7-9 supplemented with $20 \%$ by volume of $\mathrm{pH}$ 3-10 (recommended by Serva). The more effective of the separators screened in the $\mathrm{pH}$ 5-9 mixture spread the bands of higher $\mathrm{p} I$, as shown in the case of 0.2 M 6-aminocaproic acid (Fig. 2b); the others tested were $0.2 \mathrm{M}$ 4-aminobutyric acid, 3-aminopropionic acid, glycine, L-proline, DL-thre-
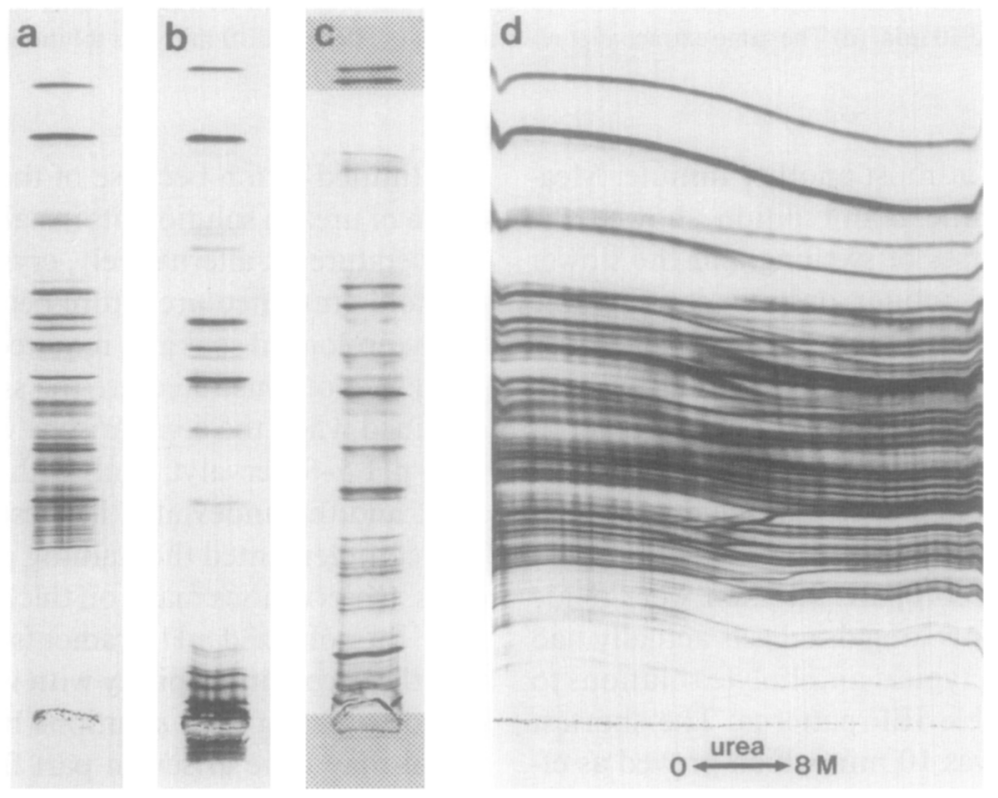

FIG. 2. IEF of newt crystallins in pH 5-9 Servalyt. The samples were free-standing in (a-c). (a) Standard rewetting solution. (b) With $0.2 \mathrm{M} 6$-aminocaproic acid added. (c) With concentration modification at the anodal end of the gel (lower gray area) extending $6.5 \mathrm{~mm}$ beyond the wick, plus concentration-thickness modification over a $5.5-\mathrm{mm}$ span (upper) starting $0.5 \mathrm{~mm}$ from the cathode wick. (d) In a transverse urea gradient; the full width of the gel is shown. 
onine, $N, N$-bis(2-hydroxyethyl)glycine, 4-morpholinepropanesulfonic acid or $N$ tris(hydroxymethyl)methyl-2-aminoethanesulfonic acid, and $10 \mathrm{mM}$ L-histidine or L-carnosine.

Because none of those additives improved separations among the crystallins of lower $\mathrm{p} I$, a third way of flattening the $\mathrm{pH}$ gradient was examined. Modification through a local increase in ampholyte concentration could be achieved by straightforward adaptation of the technique described by Låås and Olsson (9). The alternative of increasing the thickness of the layer by means of an overlaid gel (13) seemed feasible but, to avoid the casting of separate gels nearly sixfold thicker as well as the likelihood of their rewetting quite slowly, was implemented to only a limited extent as a hybrid with the preceding method. Figure $2 \mathrm{c}$ shows the widened separation of intermediate crystallins obtained when this so-called concentration-thickness modification was applied near the cathode while ordinary concentration modification was carried out at the anodal end of the gel to permit the usual sample placement. Either variant applied at the cathodal end alone distinctly improved the resolution among the bands of lowest $\mathrm{p} I$ (not shown). The techniques were simple and effective although the focusing paths often curved.

Since rewetting was well suited to the use of ampholyte mixtures containing urea, the feasibility of employing it for IEF across a urea gradient (14) was examined next. The 5-ml cassette incorporating the gel-bearing slide as one wall was filled in such a way that wetting by progressively less dense solutions proceeded up the width of the layer: the first 5-mm portion was wet with the standard solution (pH 5-9 range) containing $8 \mathrm{M}$ urea; the next 30 $\mathrm{mm}$ by an approximately linear urea gradient generated by a rotation method (15) from the limiting solutions; and the last $5 \mathrm{~mm}$ by the urea-free ampholyte mixture. To allow for the slow equilibration of urea from a large volume (12), equilibration was prolonged to $30 \mathrm{~min}$ before the solution was withdrawn in reverse and the layer prepared for IEF as usual. The gradient probably was more or less sigmoidal but the contour was not measured or likely to be very reproducible because of the erratic manner in which the rising solution traveled across the dry layer. Nevertheless, replicable and potentially informative patterns were obtained with the lens proteins (Fig. 2d).

All of the preceding studies were carried out on gels bound to microslides. Layers on polyester could not be cast as reliably but would be preferable for broad formats accommodating many samples; however, long-term storage of these dried gels was not attempted. Since a typical application might be analysis of human hemoglobins, gels bound to polyester and kept for only 1 week after drying were rewetted for IEF of a normal whole blood lysate. Previously for similar purposes, $0.2 \mathrm{M}$ each of 3-aminopropionic and 6-aminocaproic acids was added to a mainly pH 6-9 ampholyte mixture to flatten the gradient in the vicinity of $\mathrm{pH} 7$ (7). The rewetting procedure simplified surveying various ratios of the two separators in pH 5-9 Servalyt and showed that raising the concentration of 6-aminocaproic acid to 0.3 $M$ better resolved hemoglobins $A$ and $A_{1 c}$ (Fig. 3a). The components focused to equilibrium from opposing applications although starting the sample nearer the anode always gave rise to beading of hemoglobin A (one example is shown); the other protein mixtures employed in this study focused best from anodal applications.

The two-dimensional procedure for generating pH titration curves (cf. 10) was carried out as a final test of rewetted gels. Layers bound to polyester again were used but this time in the form of washed Servalyt Precotes from which the proposed 70-mm square format could be prepared readily before drying. Rewetting of these $0.1-\mathrm{mm}$-thick layers dried overnight had been found in preliminary experiments to be satisfactory for IEF purposes although the somewhat soft gels adhered to filter paper and had to be freed of excess am- 

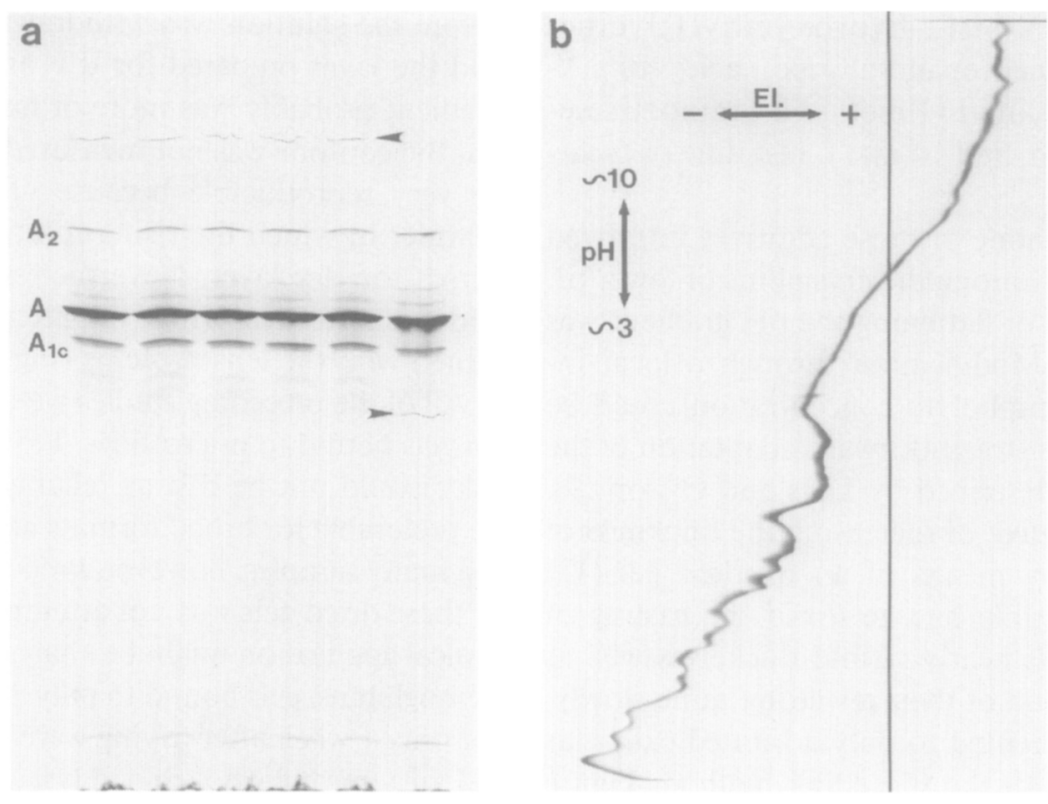

FIG. 3. Analysis of normal human hemoglobins in rewetted gels bound to polyester. (a) Replicated IFF of a whole blood lysate. The principal hemoglobins are labeled and serum proteins lie against the anode wick (bottom); arrowheads show where free-standing samples were applied. (b) Electrophoresis of a washed red blood cell lysate at right angles to an IEF gradient. Only hemoglobin A is evident; the line of sample application midway between the wicks is drawn in.

pholyte by lifting and wiping the cover film several times. However, the pattern of hemoglobin A electrophoresis transverse to an approximately pH 3-10 gradient (Fig. 3b) was reasonably similar to that obtained in 2-mmthick gels cast with ampholytes (16). Zones of diminished mobility especially in the acid range were reproducible and seemed to correspond to thinning of the gel noted at the end of the focusing step; the effect was less pronounced in firmer gels cast in the laboratory (results not shown).

The main advantages of washed and reconstituted gels over those cast ready for IEF are tolerance of higher field strengths with consequent sharpening of the focused bands, diminished distortion in the focusing patterns, and avoidance of protein artifacts induced by residual polymerizing reagents $(1,2)$; the possibility of monitoring the $\mathrm{pH}$ gradient through its fluorescent banding is one of the lesser benefits not previously exploited in ultrathin gels.
An intervening drying clearly adds considerable versatility, convenience, and economy to IEF, as is evident also from other studies along similar lines $(4,5)$, and any resulting oxidizing conditions can be circumvented (3). The need for incorporating protective additives such as glycerol before drying was found in an extensive study of gels bound to polyester (4). Although a good shelf life after simple washing and drying of layers on glass was suggested by the present work, a more demanding analysis seem warranted in view of the potential value of dried gels for standardizing IEF procedures (4).

\section{ACKNOWLEDGMENTS}

I thank Dr. Thomas G. Connelly for funds and newt lenses contributed to this project, Mr. John K. Koh of Ann Arbor Plastics, Inc., for providing developmental models of the focusing apparatus, and Dr. Robert C. Allen for access to his unpublished manuscript (5). 


\section{REFERENCES}

1. Eckersall, P. D., and Conner, J. G. (1984) Anal. Biochem. 138, 52-56.

2. Robinson, H. K. (1972) Anal. Biochem. 49, 353-366.

3. Altland, K., and Rossmann, U. (1985) Electrophoresis 6, 314-325.

4. Frey, M., Kinzkofer, A., Atta, M. B., and Radola, B. J. (1986) Electrophoresis 7, 28-40.

5. Allen, R. C., Budowle, B., Lack, P. M., and Graves, G. (1986) in Electrophoresis '86 (Dunn, M.J., ed.), VCH Publishers, Weinheim, in press.

6. Sippel, T. O. (1981) J. Histochem. Cytochem. 29, 1377-1381.

7. Cossu, G., Manca, M., Pirastru, M. G., Bullita, R., Bosisio, A. B., Gianazza, E., and Righetti, P. G. (1982) Amer. J. Hematol. 13, 149-157.
8. Caspers, M. L., Posey, Y., and Brown, R. K. (1977) Anal. Biochem. 79, 166-180.

9. Låås, T., and Olsson, I. (1981) Anal. Biochem. 114, 167-172.

10. Gunzer, G. (1984) Electrophoresis 5, 370-372.

11. Bonitati, J. (1980) J. Biochem. Biophys. Methods 2, 341-356.

12. Altland, K., Banhoff, A., Hackler, R., and Rossman, U. (1984) Electrophoresis 5, 379-381.

13. Altland, K., and Kaempfer, M. (1980) Electrophoresis $1,57-62$.

14. Hobart, M. J. (1975) in Isoelectric Focusing (Arbuthnott, J. P., and Beeley, J. A., eds.), pp. 275-280, Butterworths, London.

15. Lorentz, K. (1976) Anal. Biochem. 76, 214-220.

16. Righetti, P. G., Krishnamoorthy, R., and Gianazza, E. (1978) J. Chromatogr. 166, 455-460. 